\title{
Potential für Früherkennung von Diabetes mellitus Typ 2
}

\section{Lena Ammann ${ }^{a}$, David Fäh ${ }^{b}$}

a Bachelor of Science Ernährung und Diätetik, ${ }^{b}$ Prof. Dr. med., Facharzt für Prävention und Gesundheitswesen, Master of Public Health

In der Schweiz sind 30\% der Patienten mit Typ-2-Diabetes (DM2) unentdeckt. Durch die interprofessionelle Zusammenarbeit verschiedener Leistungserbringer, den Einbezug nicht-ärztlicher Berufsgruppen sowie durch die Lancierung von Empfehlungen hinsichtlich einer systematischen Vorgehensweise können Früherkennung und Management von DM2 verbessert werden. Dies zeigen die Ergebnisse einer Bachelorarbeit an der Berner Fachhochschule (BFH).

\section{Diabetes mellitus Typ 2: frühe Erkennung von zentraler Bedeutung}

Die Prävalenz von Diabetes mellitus Typ 2 (DM2) hat in den letzten Jahren weltweit stark zugenommen. Diese Entwicklung kann mit der immer älter werdenden Bevölkerung, verminderter körperlichen Aktivi-

\section{Résumé}

En Suisse, 30\% des personnes atteintes de diabète de type 2 (DM2) ne sont pas détectées. Un dépistage précoce des personnes présentant des valeurs de glycémie élevées permettrait de réduire les taux de morbidité et de mortalité, donc d'améliorer la qualité de vie et l'état de santé général de la population.

L'auteure principale d'une thèse de bachelor à la Haute école spécialisée bernoise (BFH) avait pour objectifs, sur la base d'une étude qualitative, d'évaluer les méthodes actuelles de dépistage précoce en Suisse, d'analyser les possibilités et les difficultés que présente son optimisation et de montrer comment son potentiel et celui de la prévention peuvent être pleinement exploités.

Les résultats mettent en évidence une grande marge de progression du dépistage précoce du DM2 en Suisse. La mise en œuvre d'un meilleur dépistage précoce du diabète est entravée par l'absence d'autorités responsables, des méthodes de diagnostic lacunaires ou l'accessibilité et les questions de financement des offres de prévention.

La collaboration interprofessionnelle de différents prestataires, l'implication de corps de métiers non médicaux et la mise en place de recommandations en vue d'une approche systématique permettraient d'améliorer le dépistage précoce et la prise en charge du DM2. tät, ungesunder Ernährung sowie der Zunahme von Adipositas begründet werden. In einer kürzlich publizierten Studie, mit subjektiv gesunden älteren Personen (älter als 60 Jahre, ohne bekannten DM2) konnte festgestellt werden, dass von der untersuchten Population $8,4 \%$ an einem DM2 und $64,5 \%$ an einem Prädiabetes (Vorstufe zum DM2) leiden, ohne es zu wissen [1]. Studien zeigen, dass in der Schweiz schätzungsweise 30\% der DM2-Betroffenen nicht als solche erkannt sind [2-4]. Dies kann auf die symptomlose Manifestation des DM2, aber auch auf das unsystematische Diabetesscreening der Schweiz zurückgeführt werden.

Durch eine rechtzeitige Früherkennung von Personen mit Zuckerstoffwechselstörungen kann die Entstehung eines DM2, mit den damit verbundenen Folgeerkrankungen, verhindert oder verzögert werden. Bereits durch eine um ein Jahr verzögerte oder ungenügende Behandlung einer gestörten Glukoseregulation steigt das Risiko, an einer Herz-Kreislauf-Krankheit (z.B. Herzinfarkt, Hirnschlag), Krebs und/oder einer Niereninsuffizienz zu erkranken [5-7].

\section{Relevanz der Prävention erkannt}

An diversen Pilotprojekten, Kampagnen und der aktuell veranlassten NCD-Strategie des Bundes wird ersichtlich, dass die Relevanz der Prävention von DM2 in der Schweiz erkannt wurde. Im Vergleich, sowohl mit etablierten Früherkennungs-Strategien nordeuropäischer Länder als auch mit den Nachbarländern Deutschland und Österreich, fällt jedoch auf, dass es in 
der Schweiz an einer stringenten Strategie für eine Vorgehensweise in der Früherkennung von DM2 fehlt [8-10]. Dementsprechend besteht in der Schweiz Potential, mit DM2 zusammenhängende Komplikationen, Erkrankungen und Kosten durch die frühzeitige Erkennung von erhöhten Blutzuckerwerten zu vermindern und damit die Lebensqualität und die allgemeine Gesundheit der Bevölkerung zu verbessern.

\section{Potential der Früherkennung in der Schweiz}

Im Rahmen einer Bachelorarbeit an der Berner Fachhochschule (BFH) hatte die Autorin zum Ziel, anhand einer qualitativen Forschungsarbeit, die Vorgehensweise der aktuellen Früherkennung in der Schweiz einzuschätzen, Chancen und Hindernisse einer verbesserten Diabetesfrüherkennung zu analysieren und aufzuzeigen, wie das Potential einer frühzeitigen Erkennung und Prävention ausgeschöpft werden kann. Mittels halbstandardisierter Leitfadeninterviews befragte sie sechs Fachpersonen aus diversen Bereichen des Gesundheitswesens. Dank der Heterogenität der Stichprobe konnten Sichtweisen und Verbesserungsvorschläge unterschiedlicher Professionen erfasst werden, wodurch eine breite Darlegung der aktuellen Lage erreicht wurde.

\section{Diabetesfrüherkennung: Verbesserungspotential in der Schweiz}

Die Ergebnisse zeigen, dass die Implementierung einer verbesserten Diabetesfrüherkennung durch einige Schwachstellen im schweizerischen Gesundheitssystem behindert wird. Sowohl bezüglich der inter- professionellen Zusammenarbeit diverser Leistungserbringer als auch bezüglich einer konsequenten methodischen und diagnostischen Vorgehensweise kann die Schweiz von ihrem internationalen Umfeld lernen [10]. Dank eines nationalen Diabetes-Registers haben skandinavische Länder wie Schweden hier eine Vorbildfunktion (siehe Box «Diabetes-Prävention» auf der nächsten Seite).

In nachfolgender Tabelle sind die bedeutendsten Schwachstellen der Diabetesfrüherkennung in der Schweiz mit deren Verbesserungsansätzen zusammengetragen.

\section{Kooperation verschiedener Akteure und Zugang zur Risikoabklärung}

Aufgrund der chronischen Überlastung vieler Praxen ist die Zusammenarbeit verschiedener Leistungserbringer zunehmend wichtig. Die zu integrierenden Leistungserbringer sollten einerseits über die nötigen Kompetenzen verfügen, um über das Krankheitsbild Auskunft geben zu können, andererseits die diagnostischen Untersuchungen durchführen können. Dabei könnte durch die vermehrte Einbindung nicht-ärztlicher Professionen das schweizerische Gesundheitssystem zugänglicher und effizienter gemacht werden Die aus den Ergebnissen hervorgehenden einzubindenden Leistungserbringer sind Berufsgruppen der Arztpraxen, der Apotheken und der Ernährungs- und Diabetesberatung. Den Apotheken kommt in diesem Zusammenhang eine wichtige Funktion als Beobachtungs-, Beratungs- und Koordinationsstelle zu, die allgemein öffentlich und ohne Terminvereinbarung zugänglich ist $[10,11,13,14]$.

Tabelle 1: Bedeutendste Schwachstellen der Diabetesfrüherkennung in der Schweiz mit deren Verbesserungsansätzen.

\begin{tabular}{|c|c|c|}
\hline Schwachstellen & Verbesserungsansätze & Ref. \\
\hline Fehlen verantwortlicher Instanzen & $\begin{array}{l}\text { Verantwortungsübernahme durch kompetente ambulante Fachstellen (Arztpraxen, Apotheken, } \\
\text { Diabetesgesellschaften) } \\
\rightarrow \text { Verantwortungsübernahme für Prävention von DM2 }\end{array}$ & {$[8,11,12]$} \\
\hline Monoprofessionalität & $\begin{array}{l}\text { Interprofessionelle Zusammenarbeit/Austausch } \\
\text { Einbezug nicht-ärztlicher Berufsgruppen } \\
\rightarrow \text { Kompetenzen diverser Berufsgruppen klären } \\
\rightarrow \text { interprofessionelles Vertrauen stärken }\end{array}$ & {$[10,11]$} \\
\hline $\begin{array}{l}\text { Zugang und Kosten von Screening } \\
\text { und präventiven Leistungen }\end{array}$ & $\begin{array}{l}\text { Öffentlicher Zugang zu Risikoabklärung und Screening-Angeboten in kompetenten Fachstellen } \\
\rightarrow \text { Leistungserbringer machen potentielle Risikopatienten auf Risikoabklärung aufmerksam } \\
\rightarrow \text { kostenloser Zugang zu Screeningleistungen }\end{array}$ & $\begin{array}{l}{[13,14,19,} \\
20]\end{array}$ \\
\hline $\begin{array}{l}\text { Mangelhafte diagnostische und } \\
\text { methodische Vorgehensweise }\end{array}$ & $\begin{array}{l}\text { 1. Risikoabklärung anhand Risikofragebogen } \\
\text { 2. Diagnosestellung mit empfohlenen Messmethoden } \\
\rightarrow \text { Verbindliche systematische Screening-Empfehlungen }\end{array}$ & [13-15] \\
\hline $\begin{array}{l}\text { Mangelhaftes Wissen über } \\
\text { - Risikofaktoren } \\
\text { - Krankheitsbild } \\
\text { - Folgeschäden }\end{array}$ & $\begin{array}{l}\text { Stärkung der Gesundheitskompetenz («Empowerment») } \\
\rightarrow \text { Sensibilisierung der Bevölkerung und der an der Früherkennung beteiligten Professionen } \\
\rightarrow \text { Schulungen zum Krankheitsbild, Folgeschäden, Nutzen frühzeitiger präventiver Interventionen }\end{array}$ & {$[6,7]$} \\
\hline
\end{tabular}




\section{Eigenverantwortung zur Risikoabklärung stärken}

Die Bevölkerung soll dazu motiviert werden, ihr Risiko für einen DM2 anhand eines unverbindlichen Risikofragebogens, basierend auf den Charakteristiken von Alter, Body Mass Index (BMI), Taillenumfang, Bluthochdruck und einer DM2-Familienanamnese, einzuschätzen und sich bei erhöhtem Risiko einer bestätigenden Kapillarblutmessung (idealerweise $\mathrm{HbA}_{1 \mathrm{c}}$ ) $\mathrm{zu}$ unterziehen [13-16].

Die Sensibilisierung für das Krankheitsbild und dessen Früherkennung spielt, sowohl in der Bevölkerung als auch bei den in der Früherkennung involvierten Berufsgruppen, eine zentrale Rolle. Das Bewusstsein darum, dass sich ein DM2 für die Betroffenen über lange Zeit symptomlos entwickelt, muss gesellschaftsübergreifend erhöht werden. Infolge der Auswirkungen des DM2, sowohl auf die Gesundheit der Bevölkerung als auch auf die Volkswirtschaft, soll der Vorteil eines frühzeitigen systematischen Screenings gefolgt von entsprechenden Ernährungs- und Bewegungsinterventionen allgemein bekannt gemacht werden $[6,7,17,18]$.

\section{Interprofessionelles Vertrauen und Zusammenarbeit}

Die Etablierung und Verbreitung einer interprofessionellen Zusammenarbeit kann nicht vom Bund erwartet werden, sondern muss von den Leistungserbringern selbst aufgebaut werden. Es ist Aufgabe jeder einzelnen Berufsgruppe, ihre eigenen Kompetenzen und Aufgabenbereiche transparent zu machen und sich in ein interprofessionelles Team zu integrieren. Alle Professionen der Arztpraxen, Apotheken, der Ernährungs- und Diabetesberatung können ihren Beitrag zur Prävention von DM2 leisten. Für die optimale Zusammenarbeit der unterschiedlichen Fachstellen bedarf es der Aufweichung von Hierarchien und der Klärung berufsspezifischer Kompetenzen und Verantwortungsbereiche. Durch einen transparenten Austausch im interprofessionellen Setting und die Erhöhung von Vertrauensverhältnissen kann eine optimierte Arbeits- und Kompetenzverteilung erfolgen, was eine Verbesserung des Ressourceneinsatzes zur Folge hat. In obligatorischen Aus- und Weiterbildungen sollen die zu integrierenden Berufsgruppen ge-

\section{Diabetes-Prävention: Was die Schweiz von Schweden lernen könnte}

- Gute Datenverfügbarkeit: Qualitätsüberwachung dank des Schwedischen Nationalen Diabetesregisters (NDR) seit 1996 - Multidisziplinäre Teams: Zusammenarbeit von Diabetesberatung, Ernährungsberatung, Podologie und Physiotherapie

- Konsequente und flächendeckende Befolgung nationaler Guidelines

- Zugang zu fachkundigem Diabetes-Management

- Individuelle Behandlungsziele: interdisziplinäres kompetentes Team erarbeitet für den Patienten personalisierte $\mathrm{Be}$ handlungsziele abhängig von Komplikationsrisiko und unter Berücksichtigung der Lebensqualität.

meinsam den Nutzen der interdisziplinären Zusammenarbeit erkennen und praktizieren lernen. Eine derartige Teamarbeit wird erleichtert, wenn die verschiedenen Teammitglieder Teil einer Organisation sind. Ansonsten muss der Arbeit an den Schnittstellen besondere Aufmerksamkeit geschenkt werden [11, 12].

\section{Konsequente einheitliche Vorgehens- weise}

In der Schweiz fehlt es an einer verbindlichen Vorgehensweise in der Früherkennung von DM2. Um in der Schweiz eine solche zu schaffen, sollten die Empfehlungen von der schweizerischen Diabetes-Gesellschaft in Zusammenarbeit mit der Schweizerischen Gesellschaft für Endokrinologie und Diabetologie (SGED) anhand der aktuellen Studienlage überarbeitet werden. Besondere Aufmerksamkeit gilt es den Bedürfnissen und Umsetzungsmöglichkeiten der einzelnen Berufsgruppen sowie den neusten Erkenntnissen in der Diagnosestellung zukommen zu lassen. Dank einer konkret empfohlenen Vorgehensweise liessen sich diagnostische und methodische Fehler beheben und Fehldiagnosen vermeiden. Dieses Vorgehen würde nicht nur Sicherheit ins System der Vorsorge für den Patienten bringen. Die konsequente Anwendung einheitlicher Kriterien geben auch den verschiedenen Akteuren die Sicherheit, die richtigen diagnostischen und therapeutischen Massnahmen zu ergreifen.

Die Literatur zu diesem Artikel findet sich online unter www.saez.ch $\rightarrow$ Aktuelle Ausgabe oder $\rightarrow$ Archiv $\rightarrow 2019 \rightarrow 8$. 